\title{
OBSERVATION OF RADIATIVE SPIN-POLARIZATION AT 60.6 GeV
}

\author{
R. Assmann, B. Dehning, M. Hildreth, J. Matheson, G. Mugnai, M. Placidi, F. Roncarolo, \\ E. Torrence, F. Sonnemann, J. Uythoven, J. Wenninger, CERN, SL Division, Geneva, Switzerland \\ A. Blondel, LPNHE Ecole Polytechnique Palaiseau, France
}

\begin{abstract}
Radiative spin-polarization has been used extensively at LEP to accurately measure the beam energy around the $\mathrm{Z}$ resonance. As the LEP physics has moved on to the $\mathrm{W}$ boson the calibration based on polarization must be extended towards higher beam energies. This is difficult as the depolarizing effects of spin resonances grow rapidly with beam energy. At LEP it has been possible for the first time to measure transverse beam polarization at $60.6 \mathrm{GeV}$. To allow a build-up of polarization the tunes and the energy were chosen accurately. A low phase advance optics was used and careful orbit correction was carried out using dynamic beam based alignment data. Harmonic spin matching was applied both in a deterministic and a novel semi-empirical way.
\end{abstract}

\section{INTRODUCTION}

Transverse spin polarization in LEP builds up spontaneously due to the Sokolov and Ternov effect [1]. Polarized beams are used in LEP since 1990 for the precise measurement of the beam energy [2]. Resonant depolarization of the initially polarized beams provides the by far most accurate method to measure the absolute energy scale. The method allowed determining the properties of the $\mathrm{Z}$ at $2 E_{\text {beam }}=91 \mathrm{GeV}$ with unsurpassed accuracy [3]. As LEP physics has moved on towards the $\mathrm{W}$ boson at around $2 E_{\text {beam }}=190 \mathrm{GeV}$, energy calibration must be extended into this range. This is difficult as the depolarizing effects of spin resonances grow rapidly with beam energy.

The LEP strategy for determining the energy scale of the $\mathrm{W}$ is to calibrate the absolute beam energy at the highest possible energy, using the highly accurate method of resonant depolarization (a minimum level of 5\% polarization is required). The scale is then extrapolated using other methods. The final error on the energy scale for the $\mathrm{W}$ depends on the range that must be extrapolated. In 1998 polarization was for the first time established in LEP at $60.6 \mathrm{GeV}$, allowing a more accurate energy calibration of LEP.

\section{OVERVIEW ON POLARIZATION OPTIMIZATION IN LEP}

The polarization build-up in a $e^{+} e^{-}$storage ring is characterized by a build-up time $\tau_{p}$. The build-up time is inversely proportional to the fifth power of the beam energy; polarization build-up is therefore strongly enhanced with increasing beam energy. Concurrently unavoidable imperfections cause depolarizing effects. They are characterized by a depolarization time $\tau_{d}$. The asymptotic polarization $P_{\infty}$ describes the equilibrium between the two processes:

$$
P_{\infty}=\frac{92.4 \%}{1+\frac{\tau_{p}}{\tau_{d}}}=\frac{92.4 \%}{1+\alpha^{2} E_{\text {beam }}^{2}}
$$

The term $\tau_{p} / \tau_{d}$ has been parameterized by an energyindependent imperfection term $\alpha$ and the beam energy $E_{\text {beam. }}$. The given scaling law applies only for the most optimistic scenario, the so-called linear regime of polarization. LEP, for higher energies, operates in the so-called higher-order regime and the decrease in equilibrium polarization is much steeper with increasing energy. The theory of polarization for high energy $e^{+} e^{-}$storage rings is summarized in [4] and is evaluated for LEP in [5].

The process of polarization optimization in LEP trys to maximize the degree of spin-polarization in LEP. It is crucial to minimize the imperfection term $\alpha$. The optimization process at LEP can be summarized as follows:

- Problem: Imperfections in beam position monitors (BPM's) and quadrupoles. Solution: Hardware alignment, dynamic beam-based alignment.

- Problem: Orbit offsets and dispersion due to residual imperfections. Solution: Orbit correction, knob tuning, dispersion-free steering, low phase advance optics.

- Problem: Excitation of depolarizing resonances due to residual orbit offsets and dispersion. Solution: Energy and tune scan to sit in between resonances, deterministic and semi-empirical harmonic spin matching.

A strong effort is invested at LEP in order to improve the chances for polarized beams at highest beam energies.

\subsection{Accelerator alignment and optics}

Vertical quadrupole misalignments are the main origin of depolarizing effects. They are minimized with a yearly realignment of all LEP quadrupoles. The vertical offsets of the BPM's with respect to the center of the quadrupoles is determined via a dynamic beam-based alignment technique [6]. The effects of the residual quadrupole misalignments on the beam orbit are minimized with a low phase advance optics.

\subsection{Basic orbit and energy setup}

The residual imperfections cause orbit offsets and dispersion. Standard MICADO orbit correction is used to minimize the vertical orbit especially in the arcs of the storage ring. Special attention must be put on eliminating any 
$\pi$-bump in the accelerator. A coupling correction is performed via special coupling knobs. A dispersion-free orbit correction reduces dispersion and allows to find additional $\pi$-bumps. This method has been tested in 1998 and will be used routinely starting from 1999.

Depolarization is a resonant process. Therefore special care must be taken to avoid depolarizing resonances. The beam energy must be adjusted such that the working point has a maximum distance to all important spin resonances. The required accuracy in the setting of the beam energy is on the order of $\pm 10 \mathrm{MeV}$ for high energies. Nuclear magnetic resonance devices are used to extrapolate the beam energy from a measurement at about $45 \mathrm{GeV}$.

\subsection{Harmonic Spin-Matching}

Depolarization for high energy storage rings is mainly driven by the residual vertical orbit deviations $y_{i}(i=1, N)$ in the $N$ quadrupoles. The Fourier components of the orbit deviations are important:

$$
\begin{aligned}
a_{k} & =\frac{1}{\pi} \sum_{i=1}^{N} y_{i} \cos \left[k \alpha_{i}\right] \delta \alpha_{i} \\
b_{k} & =\frac{1}{\pi} \sum_{i=1}^{N} y_{i} \sin \left[k \alpha_{i}\right] \delta \alpha_{i}
\end{aligned}
$$

Here, $k$ is the harmonic number, $\alpha_{i}$ is the integrated bending angle up to quadrupole $i$ (running from 0 to $2 \pi$ ) and $\delta \alpha_{i}$ is the change in bending angle for quadrupole $i . a_{k}$ is the "cosine" strength and $b_{k}$ the "sine" strength of harmonic $k$. $\tau_{p} / \tau_{d}$ can then be written as:

$$
\frac{\tau_{p}}{\tau_{d}}=\kappa^{\prime} \nu^{2} \sum_{k} \frac{a_{k}^{2}+b_{k}^{2}}{(\nu-k)^{4}}
$$

Here, $\nu$ is the spin tune $\left(\nu=E_{\text {beam }} / 440.65 \mathrm{MeV}\right)$ and $\kappa^{\prime}$ is some constant. It is seen that only the two closest harmonics $k_{0}=\operatorname{Int}(\nu), k_{1}=\operatorname{Int}(\nu)+1$ are important. $\nu$ is put close to an half-integer for LEP. The polarization is then:

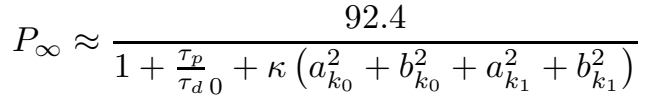

Note that for a given energy the spin tune and $\kappa^{\prime}$ terms have been put into the constant $\kappa$. The term $\left(\tau_{p} / \tau_{d}\right)_{0}$ takes into account depolarizing effects from less important sources.

Harmonic Spin Matching (HSM) is performed by introducing perturbations $\hat{a}_{k_{0}}, \hat{b}_{k_{0}}, \hat{a}_{k_{1}}$ and $\hat{b}_{k_{1}}$ such that the harmonics due to vertical orbit offsets are cancelled [7]. Special orbit bumps are used in LEP to generate the harmonics [8]. The bumps are chosen such that changes in dispersion, coupling etc. are minimized and additional depolarization is not introduced. The bumps were re-optimized in 1998. The HSM consists in minimizing the following system:

$$
\begin{gathered}
\frac{92.4 \%}{P_{\infty}} \approx 1+\frac{\tau_{p}}{\tau_{d} 0}+\kappa\left[\left(a_{k_{0}}-\hat{a}_{k_{0}}\right)^{2}+\left(b_{k_{0}}-\hat{b}_{k_{0}}\right)^{2}+\right. \\
\left.\left(a_{k_{1}}-\hat{a}_{k_{1}}\right)^{2}+\left(b_{k_{1}}-\hat{b}_{k_{1}}\right)^{2}\right]
\end{gathered}
$$

Two different approaches have successfully been applied.
A) A fully empirical approach, where each of the four harmonics is scanned in turn while polarisation is measured. For each bump the setting where polarisation is maximum is kept. This is the method applied at PETRA [9] and HERA [10], with several (typically 5) settings for each harmonic. The most important difficulties in applying this empirical method to LEP are: 1) The long polarization time $\left(\tau_{p}=300\right.$ minutes at $45 \mathrm{GeV}, 80$ minutes at $\left.\left.60 \mathrm{GeV}\right) .2\right)$ The low degree of polarization. 3) The limited accuracy in the polarisation measurement $(\approx 0.8 \% /$ minute. At high energies the initial LEP polarisation is $1-2 \%$. A polarization of $1 \%$ corresponds to $\tau_{p} / \tau_{d} \approx 100$. Halving $\tau_{p} / \tau_{d}$ increases the polarization to about $2 \%$. This is difficult to measure.

B) The deterministic spin-matching, where the orbit data are used to determine the Fourier harmonics directly. This method is ideal if precision is sufficient, and has proven very successfull at the $Z$ peak [11]. However, as energy increases the strength of depolarizing resonances increases and the precision required is very high. The quality of the orbit measurement has been improved recently with the dynamic beam-based alignment [6]. The deterministic method does give a good starting point and an estimate of the location of the overall optimum, but is often insufficient by itself at high energies to provide optimal results.

The two established methods of HSM are not sufficient for LEP at high energies. A semi-empirical method has therefore been applied in 1998. It can be noticed that Eq. 6 depends on only five parameters: Four optimal settings $\hat{a}_{k_{0}}$, $\hat{b}_{k_{0}}, \hat{a}_{k_{1}}, \hat{b}_{k_{1}}$ and the residual depolarization $\left(\frac{\tau_{p}}{\tau_{d}}\right)_{0}$. The semi-empirical HSM procedure consists in measuring the polarization for five different settings of the HSM bumps and then performing a fit of the observed polarization levels to Eq. 6. We now take as an example the case where the starting polarization level is around $2 \%$. This means that the total depolarization term $\frac{\tau_{p}}{\tau_{d}} \simeq 50$. If one hopes to reach a polarization level of $6 \%$, this means that $\frac{\tau_{p}}{\tau_{d}}$ is around 15 . The harmonics to be corrected contribute 35 , i.e. typically 9 per harmonic; the harmonics are typically off by 3 units. This sets the scale for the changes to make to the bumps in order to scan them efficiently. When all four considered HSM bumps have been scanned, a total of five points is available. A fit to the polarization data gives a new optimum. One can then set oneself to the predicted optimum, and repeat the procedure.

\section{MEASUREMENT AT $60.6 \mathrm{GeV}$}

In September 1998 sufficient polarization for energy calibration was established for the first time at $60.6 \mathrm{GeV}$. A careful application of the optimization procedures provided a polarization of $2-3 \%$ after deterministic but before semiempirical HSM. At this time the process of semi-empirical harmonic spin matching was applied. Table 3 summarizes the settings $\hat{a}_{k_{0}}(\cos ), \hat{b}_{k_{0}}(\sin ), \hat{a}_{k_{1}}(\cos ), \hat{b}_{k_{1}}(\sin )$ for harmonics $k_{0}=137$ and $k_{1}=138$. Figure 1 shows the observed response in polarization and the fits of the asymptotic polarization level. The fit results are tabulated in Ta- 
ble 3. Data were taken with the $60 / 60$ degree optics, no low beta and experimental solenoids off.

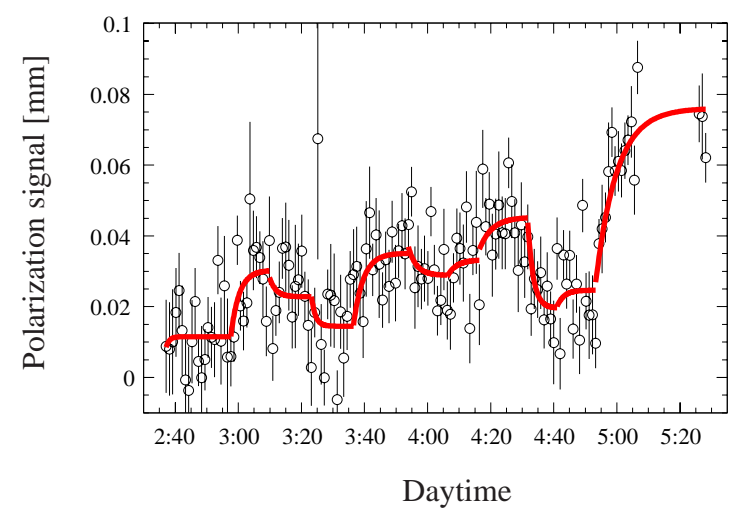

Figure 1: Polarization data from 21 September 1998. Shown is the measured polarization signal. Superimposed is the result of the polarization fits. The horizontal axis is the daytime.

Table 1: Results of polarization fits for different harmonic components. The last line shows the result of the global fit over all measurements.

\begin{tabular}{|c|c|c|c|c|c|}
\hline & \multicolumn{4}{|c|}{ HSM bumps settings } & Fit results \\
\hline $\begin{array}{c}\text { Time } \\
\text { hr:min }\end{array}$ & $\begin{array}{r}137 \\
(\cos )\end{array}$ & $\begin{array}{r}137 \\
(\sin )\end{array}$ & $\begin{array}{r}138 \\
(\cos )\end{array}$ & $\begin{array}{c}138 \\
(\sin )\end{array}$ & $\overline{P_{\infty}(\%)}$ \\
\hline $02: 35$ & 0.0 & $\overline{0.0}$ & $\overline{0.0}$ & $\overline{0.0}$ & $1.15 \pm 0.23$ \\
\hline $02: 58$ & 2.0 & 0.0 & 0.0 & 0.0 & $3.03 \pm 0.37$ \\
\hline 03:10 & 2.0 & 0.0 & 2.0 & 0.0 & $2.28 \pm 0.30$ \\
\hline $03: 23$ & 2.0 & 0.0 & 2.0 & 2.0 & $1.45 \pm 0.27$ \\
\hline $03: 36$ & 2.0 & 2.0 & 2.0 & 0.0 & $3.51 \pm 0.28$ \\
\hline 03:54 & 2.0 & 2.0 & 2.0 & -2.0 & $2.88 \pm 0.25$ \\
\hline 04:06 & 2.0 & 2.0 & 2.0 & 0.0 & $3.33 \pm 0.40$ \\
\hline 04:16 & 4.0 & 2.0 & 2.0 & 0.0 & $4.53 \pm 0.34$ \\
\hline 04:33 & 6.0 & 2.0 & 2.0 & 0.0 & $1.86 \pm 0.27$ \\
\hline 04:41 & 3.0 & 4.0 & 2.0 & 0.0 & $2.66 \pm 0.35$ \\
\hline $04: 53$ & 3.0 & 2.0 & 0.6 & -0.6 & $7.69 \pm 0.36$ \\
\hline
\end{tabular}

The last line of Table 3 shows the result from the global fit of the optimal harmonic components. The fitted harmonics were applied and polarization rose to $(7.7 \pm 0.4) \%$. This level of polarization was comfortably above the $5 \%$ as required for energy calibration by resonant depolarization. The process of semi-empirical HSM required about 1.5 hours, being significantly faster than empirical HSM.

The maximum measured polarization levels in LEP are shown in Figure 2 as a function of beam energy. The measurement at $60.6 \mathrm{GeV}$ is higher than the polarization observed at $55 \mathrm{GeV}$, demonstrating the excellent setup at $60.6 \mathrm{GeV}$. The observed decrease in polarization from $45 \mathrm{GeV}$ to $60.6 \mathrm{GeV}$ can be fully explained from the higher-order polarization theory [5].

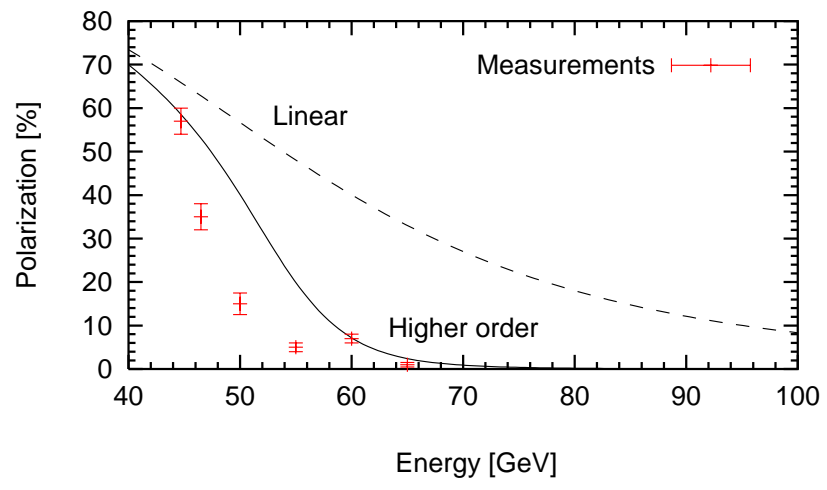

Figure 2: Measured maximum polarization in LEP for different energies. The data is compared to the higher-order (solid curve) and the linear (dashed curve) prediction, assuming equal residual imperfections after correction.

\section{CONCLUSION}

Highly accurate energy calibration at LEP requires transverse spin-polarization of at least $5 \%$ up to the highest beam energies. Polarization drops sharply with increasing beam energy. Therefore extensive optimizations of the storage ring are required in order to extend polarization to the highest beam energies. The quadrupoles are aligned and a special optics is being used; the tunes and the beam energy are chosen accurately to avoid spin resonances; a careful orbit correction is carried out using dynamic beam based alignment data; harmonic spin matching is applied both in a deterministic and a novel semi-empirical way. The optimization allowed for the first time to establish a clear polarization signal at $60.6 \mathrm{GeV}$. After optimization a polarization degree of $(7.7 \pm 0.4) \%$ was measured. This is, in terms of residual machine imperfections, equivalent to the polarization record of $57 \%$ at $44.7 \mathrm{GeV}$. In 1999 it will be tried to extend polarization and energy calibration to $70 \mathrm{GeV}$ or even higher [5].

\section{REFERENCES}

[1] A.A. Sokolov and I.M. Ternov, Sov. Phys. Dokl. 8(1964)1203.

[2] L. Arnaudon et al, Z. Phys. C66(1995)45.

[3] R. Assmann et al, Eur. Phys. J. C6(1999)187-223.

[4] Ya.S. Derbenev, A.M. Kontratenko and A.N. Skrinsky, Part. Acc. 1979, Vol. 9, pp. 247-266.

[5] R. Assmann, these proceedings.

[6] B. Dehning et al., CERN SL/98-38-BI.

[7] R. Rossmanith and R. Schmidt, Nucl. Instr. Meth. A236(1985)231.

[8] R. Assmann et al., CERN SL/94-08(AP).

[9] H.D. Bremer et al, DESY-M 82-026(1982).

[10] D.P. Barber et al, DESY 93-038.

[11] R. Assmann, et al., CERN SL/94-62. 